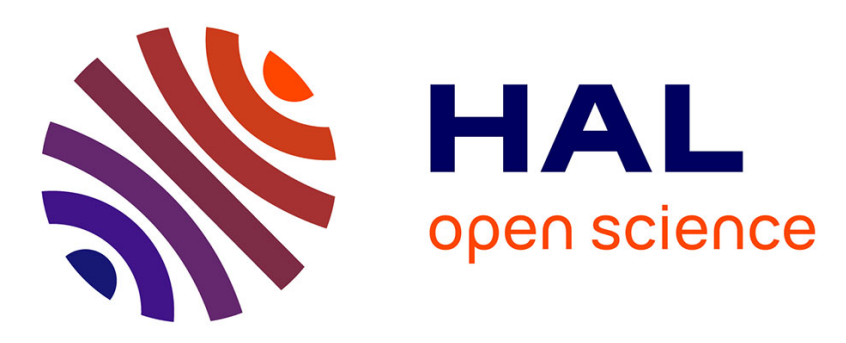

\title{
Impact of array calibration on RFI mitigation
}

Gregory Hellbourg, Karim Abed-Meraim, Rodolphe Weber

\section{To cite this version:}

Gregory Hellbourg, Karim Abed-Meraim, Rodolphe Weber. Impact of array calibration on RFI mitigation. Conference: 24th European Signal Processing Conference (EUSIPCO) Location: Budapest, HUNGARY Date: AUG 28-SEP 02, 2016, Aug 2016, Budapest, Hungary. p 85-89, 10.1109/EUSIPCO.2016.7760255 . insu-01467269

\section{HAL Id: insu-01467269 \\ https://hal-insu.archives-ouvertes.fr/insu-01467269}

Submitted on 3 Jul 2020

HAL is a multi-disciplinary open access archive for the deposit and dissemination of scientific research documents, whether they are published or not. The documents may come from teaching and research institutions in France or abroad, or from public or private research centers.
L'archive ouverte pluridisciplinaire HAL, est destinée au dépôt et à la diffusion de documents scientifiques de niveau recherche, publiés ou non, émanant des établissements d'enseignement et de recherche français ou étrangers, des laboratoires publics ou privés. 


\title{
Impact of array calibration on RFI mitigation
}

\author{
Gregory Hellbourg*, Karim Abed-Meraim ${ }^{\dagger}$, Rodolphe Weber ${ }^{\dagger \ddagger}$ \\ ${ }^{*}$ CSIRO Astronomy and Space Science, Sydney, Australia \\ ${ }^{\dagger}$ Laboratoire PRISME, Université d'Orléans, Orléans, France \\ ${ }^{\ddagger}$ Station de radioastronomie de Nançay, Observatoire de Paris, CNRS/INSU, Nançay, France
}

\begin{abstract}
Phased array radio telescopes allow for the filtering of Radio Frequency Interference (RFI) in the spatial domain. Spatial filters are advantageous in radio astronomy when the separation between RFI and astronomical sources cannot be made in the time or frequency domains.

Consequently, the mitigation of the RFI relies on the quality of its spatial signature vector (SSV) estimation. The latter depends on the array calibration information which is investigated in this work. More precisely, by using the Cramér-Rao Bound (CRB) tool, we evaluate the astronomical source power estimation error variance in presence of RFI for different array calibration scenarios corresponding to perfectly calibrated, direction-independent uncalibrated and direction-dependent uncalibrated array cases. In addition, we consider in this study the case where only the data covariance information is available and investigate the loss of performance due to the missing data with respect to different system parameters.
\end{abstract}

Index Terms-Cramér-Rao bound, RFI mitigation, Array processing, Radio astronomy

\section{INTRODUCTION}

Phased array telescopes are beneficial to radio astronomy [1]. Advantages compared to single dish instruments include the ability to perform high resolution observations, steering at independent directions simultaneously and designing beam and side lobes at will. Although the collecting area - and therefore the sensitivity - of such instrument is usually reduced, recent developments of phased array feeds [2], [3], [4] for dish radio telescopes are overcoming this limitation. Another major advantage of phased arrays is the possibility to process the collected data in the spatial domain. Particularly, they allow the use of spatial filters for blindly isolating sources of interest from interfering sources when a dissociation in the time and frequency domains is not possible. This feature is relevant for radio astronomy due to the increasing occupancy the electromagnetic spectrum by man-made sources [5], [6].

Various spatial filtering strategies have been investigated for Radio Frequency Interference (RFI) mitigation for radio astronomy, most of them relying on data and instrumental assumptions and prior information concerning the source of RFI [7], [8]. The orthogonal projector, for instance, is able to perform deep nulls in the direction of an interferer given its spatial signature vector (SSV) [9]. The SSV describes the contribution of the interfering signal to the array data. This quantity cannot be determined without prior information regarding the relative location of the source of RFI and an accurate array calibration, and requires therefore to be estimated from the collected data. The SSV estimation quality is the limiting factor of a projector performance.
Various RFI SSV estimators for phased arrays radio telescopes have been suggested. These estimators are based on features allowing a separation between interfering signals, astronomical sources and system noise. Features include Interference-to-Noise Ratio (covariance matrix) [9], cyclostationarity (cyclic covariance matrix) [10], non-circularity (pseudo-covariance matrix) [11], finite bandwidth (time-lagged covariance matrix) [12] or spatial location information (independent reference antenna) [13]. The behavior and performance of these estimators depends on the considered data model.

This article provides a comparative study of the SSV estimation performance and related astronomical source power estimation error as a function of the array calibration [14]. In other words, we investigate the impact of the calibration on the RFI mitigation performance. Since most communication signals are cyclostationary, we considered a Gaussian cyclic RFI signal and compared the corresponding CRB expressions when accessing the "full data" information and for the "missing data" case using the covariance information only.

\section{DATA MODELS AND SSV ESTIMATORS}

\section{A. RFI subspace}

The propagation delay of a far-field narrow band interfering signal $r(t)$ between a reference point in space and the $k^{\text {th }}$ element of an $M$-elements phased array is expressed as a signal phase shift:

$$
\mathbf{a}_{r_{k}}=e^{i \frac{2 \pi}{\lambda} \mathbf{c}_{k} \mathbf{s}_{r}} \quad k=1 . . M
$$

where $i=\sqrt{-1}, \lambda$ is the observed wavelength, $\mathbf{c}_{k}=\left[\begin{array}{ll}x_{k} & y_{k}\end{array}\right]$ are the coordinates of the $k^{\text {th }}$ array element in an arbitrary referential centered on the reference point (considered here as being the first sensor position, i.e. $x_{1}=y_{1}=0$ ), and $\mathbf{s}_{r}=\left[\begin{array}{ll}\sin \theta_{r} \cos \phi_{r} & \sin \theta_{r} \sin \phi_{r}\end{array}\right]^{T}$ with $\left(\theta_{r}, \phi_{r}\right)$ the elevation $\left(\left[0^{\circ}, 90^{\circ}\right]\right)$ and azimuth $\left(\left[0^{\circ}, 360^{\circ}[)\right.\right.$ coordinates of the source of interference, respectively, expressed in the same referential. $(.)^{T}$ is the transpose operator.

The RFI SSV $\mathbf{a}_{r}$ is the $M \times 1$ vector containing the phases for all array elements. This vector is a basis for the onedimensional RFI subspace. When an unbiased estimate $\hat{\mathbf{a}}_{r}$ of $\mathbf{a}_{r}$ is available, the subspace generated by this estimate is projected out of the array data vector space through, for instance, the orthogonal projection operator $\mathbf{P}_{\hat{\mathbf{a}}_{r}}$ defined as [15]: 


$$
\mathbf{P}_{\hat{\mathbf{a}}_{r}}=\mathbf{I}-\hat{\mathbf{a}}_{r}\left(\hat{\mathbf{a}}_{r}^{H} \hat{\mathbf{a}}_{r}\right)^{-1} \hat{\mathbf{a}}_{r}^{H}
$$

with $\mathbf{I}$ the identity matrix and $(.)^{H}$ the Hermitian transpose.

Such a projector is used to mitigate the interference signal in order to properly estimate the astronomical source power in the steering direction ${ }^{1}$. The estimation error of this SSV (depending on the array calibration) would affect the desired estimation performance as will be detailed next.

\section{B. Array data model}

The output of a phased array radio telescope subject to an astronomical source and an interfering signal is expressed as follows:

$$
\mathbf{x}(t)=\mathbf{D}_{r} \mathbf{a}_{r} r(t)+\mathbf{D}_{s} \mathbf{a}_{s} s(t)+\mathbf{n}(t)
$$

- $\mathbf{x}(t)$ is the $M \times 1$ array output vector,

- $r(t)=a(t) e^{i(\omega t+\psi)}$ the non-circular cyclostationary amplitude modulated interfering signal with $a(t) \sim$ $\mathcal{N}\left(0, \sigma_{r}^{2}\right)$ a real stationary Gaussian random amplitude signal with independent and identically distributed (i.i.d) samples ${ }^{2}$,

- $s(t) \sim \mathcal{N C}\left(0, \sigma_{s}^{2}\right)$ is the white stationary i.i.d. complex Gaussian random astronomical source signal. A main objective in radio astronomy would be to properly estimate the signal power $\sigma_{s}^{2}$ in the chosen steering direction $\left(\theta_{s}, \phi_{s}\right)$.

- $\mathbf{a}_{s}$ is the $M \times 1$ astronomical source SSV such that $\mathbf{a}_{s_{k}}=e^{i \frac{2 \pi}{\lambda} \mathbf{c}_{k} \mathbf{s}_{s}}$ with $\mathbf{s}_{s}=\left[\begin{array}{ll}\sin \theta_{s} \cos \phi_{s} & \sin \theta_{s} \sin \phi_{s}\end{array}\right]^{T}$, $\left(\theta_{s}, \phi_{s}\right)$ being the known elevation and azimuth coordinates of the steering direction corresponding to the desired astronomical source,

- $\mathbf{n}(t) \sim \mathcal{N C}\left(\mathbf{0}, \sigma_{n}^{2} \mathbf{I}\right)$ is the white stationary random system noise vector with i.i.d. complex Gaussian distribution.

The three calibration scenarios considered are:

- Perfectly calibrated: $\mathbf{D}_{r}=\mathbf{D}_{s}=\mathbf{I}$.

- Direction-independent (DI-)uncalibration: $\mathbf{D}_{r}=\mathbf{D}_{s}=$ $\operatorname{diag}(\mathbf{u})$ where $\mathbf{u}$ is a normalized uncalibration vector such that $\mathbf{u}_{k}=g_{k} e^{i \phi_{k}}$ with $u_{1}=1$ and $\operatorname{diag}($.$) is the$ vector-to-diagonal matrix operator.

- Direction-dependent (DD-)uncalibration: $\mathbf{D}_{r}=\operatorname{diag}\left(\mathbf{u}_{r}\right)$ and $\mathbf{D}_{s}=\operatorname{diag}\left(\mathbf{u}_{s}\right)$ where $\mathbf{u}_{r_{k}}=g_{r_{k}} e^{i \phi_{r_{k}}}, \mathbf{u}_{s_{k}}=$ $g_{s_{k}} e^{i \phi_{s_{k}}}, \mathbf{u}_{r_{1}}=\mathbf{u}_{s_{1}}=1$ and $\mathbf{u}_{r}$ and $\mathbf{u}_{s}$ are normalized. We also further assume $\mathbf{a}_{r}=\mathbf{a}_{s}=\overrightarrow{\mathbf{1}}_{M}$ in the DDuncalibration scenario, where $\overrightarrow{\mathbf{1}}_{M}$ is the $M \times 1$ vector made of ones only, as the sources spatial coordinates are not relevant in this case anymore.

Consider the extended array output vector $\tilde{\mathbf{x}}(t)=$ $\left[\mathbf{x}(t)^{T} \mathbf{x}(t)^{H}\right]^{T} . \tilde{\mathbf{x}}(t)$ is a random i.i.d. complex Gaussian vector distributed as $\tilde{\mathbf{x}}(t) \sim \mathcal{N C}(\mathbf{0}, \mathbf{R})$ with

\footnotetext{
${ }^{1}$ This is for skymap evaluation.

${ }^{2}$ More generally, one can assume $a(t)$ to be non-circular complex Gaussian random process.
}

$$
\mathbf{R}(t)=\left[\begin{array}{cc}
\mathbf{R}_{\mathbf{x x}} & \mathbf{R}_{\mathbf{x x}^{T}}(t) \\
\mathbf{R}_{\mathbf{x x}^{T}}^{*}(t) & \mathbf{R}_{\mathbf{x x}^{H}}^{*}
\end{array}\right]
$$

$(.)^{*}$ is the complex conjugate operator and

$$
\begin{aligned}
& \mathbf{R}_{\mathbf{x x}^{H}}=\sigma_{r}^{2} \mathbf{a}_{r_{\text {uncal }}} \mathbf{a}_{r_{\text {uncal }}}^{H}+\sigma_{s}^{2} \mathbf{a}_{s_{\text {uncal }}} \mathbf{a}_{s_{\text {uncal }}}^{H}+\sigma_{n}^{2} \mathbf{I} \\
& \mathbf{R}_{\mathbf{x x}^{T}}(t)=\sigma_{r}^{2} e^{i 2(\omega t+\psi)} \mathbf{a}_{r_{\text {uncal }}} \mathbf{a}_{r_{\text {uncal }}^{T}}^{T}
\end{aligned}
$$

where for notation simplicity, we introduce $\mathbf{a}_{r_{\text {uncal }}}=\mathbf{D}_{r} \mathbf{a}_{r}$ and $\mathbf{a}_{s_{\text {uncal }}}=\mathbf{D}_{s} \mathbf{a}_{s}$.

\section{CRAmÉR-RAo Bound Analysis}

Various estimation performance analysis for phased array systems have been conducted for signal parameters estimation. A significant amount of studies addressed the problem of source localization [16]. This information being either unavailable or irrelevant for uncalibrated arrays, the present paper addresses the RFI SSV estimation performance and related signal power estimation quality.

The CRB for a given data model parameter expresses the minimum variance expected from an unbiased estimator of this parameter. This quantity allows a relevant estimators comparison and optimum signal processing performance evaluation.

\section{A. Full data information case}

The CRB of the Gaussian data model considered in Equation (3) is contained in the diagonal elements of the CRB matrix $\mathbf{P}_{\mathrm{CRB}}$ defined as [16]:

$$
\left[\mathbf{P}_{\mathrm{CRB}}^{-1}\right]_{i, j}=\frac{1}{2} \sum_{t=0}^{T-1} \operatorname{tr}\left(\mathbf{R}^{-1}(t) \frac{\partial \mathbf{R}}{\partial \theta_{i}}(t) \mathbf{R}^{-1}(t) \frac{\partial \mathbf{R}}{\partial \theta_{j}}(t)\right)
$$

where $T$ is the sample size and the parameter vector $\theta$ is data model-dependent and takes the following form:

- Calibrated model:

$$
\theta=\left[\theta_{r}, \phi_{r}, \sigma_{r}^{2}, \omega, \psi, \sigma_{s}^{2}, \sigma_{n}^{2}\right]^{T}
$$

- Direction-independent uncalibrated model:

$$
\begin{aligned}
\theta= & {\left[\theta_{r}, \phi_{r}, \sigma_{r}^{2}, \omega, \psi, \sigma_{s}^{2}, \sigma_{n}^{2} \ldots\right.} \\
& \left.\left\{g_{k}\right\}_{k=2 . . M},\left\{\phi_{k}\right\}_{k=2 . . M}\right]^{T}
\end{aligned}
$$

- Direction-dependent uncalibrated model:

$$
\begin{aligned}
\theta= & {\left[\left\{g_{r_{k}}\right\}_{k=2 . . M},\left\{\phi_{r_{k}}\right\}_{k=2 . . M}, \sigma_{r}^{2}, \omega, \psi \ldots\right.} \\
& \left.\left\{g_{s_{k}}\right\}_{k=2 . . M},\left\{\phi_{s_{k}}\right\}_{k=2 . . M}, \sigma_{s}^{2}, \sigma_{n}^{2}\right]^{T}
\end{aligned}
$$

The covariance matrix derivatives are straightforward, and this analysis is limited to numerical evaluation of the parameters CRB. In particular, we compare in the sequel the minimal error variance given by CRB $\left\{\sigma_{s}^{2}\right\}$ and CRB $\left\{\left|\mathbf{a}_{r_{\text {uncal }}^{H}}^{H} \hat{\mathbf{a}}_{r_{\text {uncal }}} / M-1\right|^{2}\right\}$ (see [17] for definition) for the different scenarios mentioned previously.

The simulation conducted involves a flat array made of 10 antennas normally distributed over a circular area of $80 \mathrm{~m}$ of diameter and observing at frequency $f_{0}=150 \mathrm{MHz} .100$ 
data samples are generated. The astronomical source power and system noise power are unitary $\left(\sigma_{s}^{2}=\sigma_{n}^{2}=1\right.$, Signalto-Noise Ratio SNR $=0 \mathrm{~dB}$ ), and the uncalibration gains and phases for $\mathbf{D}_{r}$ and $\mathbf{D}_{s}$ are randomly and uniformly distributed between $[1-5 \%, 1+5 \%]$ and $[0,2 \pi]$, respectively ${ }^{3}$. The RFI carrier parameters $\omega$ and $\psi$, as well as the azimuth angles $\phi_{r}$ and $\phi_{s}$, are randomly generated from a uniform distribution over $[0,2 \pi]$, the elevation angles $\theta_{r}$ and $\theta_{s}$ are generated from a uniform distribution over $\left[0, \frac{\pi}{2}\right]$.

The "square" data points on Figure 1 show, in this case study, the CRB of parameter $\sigma_{s}^{2}$ in the three calibration scenarios according to the Interference-to-Signal Ratio (ISR) defined as

$$
\mathrm{ISR}=10 \log _{10}\left(\sigma_{r}^{2} / \sigma_{s}^{2}\right)
$$

The calibrated scenario achieves the best performance. The $\mathrm{CRB}$ in the DI-scenario converges towards a similar value as in the calibrated scenario when the RFI dominates the data model (ISR $>10 \mathrm{~dB}$ ), allowing a more accurate RFI localization and therefore mitigation. No prior knowledge regarding the astronomical source being considered in the DD-uncalibration, the performance of a statistically optimum astronomical source power estimator appears to be independent of the ISR, with an approximate $5 \mathrm{~dB}$ performance loss compared to the calibrated scenario.

The Hermitian angle $\Theta_{H}$ between the RFI subspace $\left(\operatorname{span}\left\{\mathbf{a}_{r}\right\}\right)$ and the astronomical subspace $\left(\operatorname{span}\left\{\mathbf{a}_{s}\right\}\right)$ describes a geometrical angular separation between these subspaces and is defined as [18]:

$$
\Theta_{H}=\left|\mathbf{a}_{r}^{H} \mathbf{a}_{s}\right| /\left\|\mathbf{a}_{r}\right\| /\left\|\mathbf{a}_{s}\right\|
$$

with $\|\cdot\|$ the vector Euclidean norm.

An analysis of the CRB for the astronomical source power at ISR $=0 \mathrm{~dB}$ and $\mathrm{SNR}=0 \mathrm{~dB}$ according to the Hermitian angle is shown on Figure 2 ("square" data points). The separation between the RFI and the astronomical subspaces does not seem to influence the CRB as long as the latter is larger than the resolution limit. The calibrated scenario offers again the best performance. An approximate $3 \mathrm{~dB}$ performance loss is noticed between the DI-uncalibrated and calibrated scenarios, and about $5 \mathrm{~dB}$ between the DD-uncalibrated and calibrated scenarios.

The "square" points on Figure 3 show the CRB of the RFI $\mathrm{SSV} \mathbf{a}_{r_{\text {uncal }}}$ according to the ISR at SNR $=0 \mathrm{~dB}$. Whereas the vector estimation error keeps decreasing for ISR $>20 \mathrm{~dB}$, the astronomical source estimation error reaches a plateau as shown on Figure 1. Remarkably, this plateau reaches the same value for both calibrated and DI-uncalibrated scenarios while a constant offset (about $20 \mathrm{~dB}$ ) in performance is noted RFI SSV estimation error variance. The $\approx 4 \mathrm{~dB}$ performance loss between the calibrated and the DD-uncalibated scenarios for $\operatorname{CRB}\left\{\sigma_{s}^{2}\right\}$ is also observed for CRB $\left\{\mathbf{a}_{r_{\text {uncal }}}\right\}$.

\footnotetext{
${ }^{3}$ For comparison fairness, we normalize these matrices so that the SSVs are of constant norm equal to $M$.
}

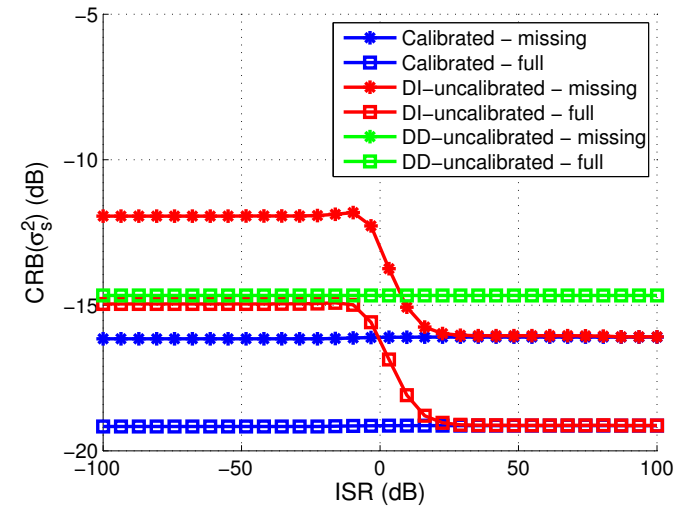

Fig. 1. Evolution of the CRB of parameter $\sigma_{s}^{2}$ at $\mathrm{SNR}=0 \mathrm{~dB}$ according to the ISR (in dB) for the calibrated scenario (blue), DI-uncalibrated scenario (red) and DD-uncalibrated scenario (green). The "full data" case is represented by squares, whereas the "missing data" case is represented by stars.

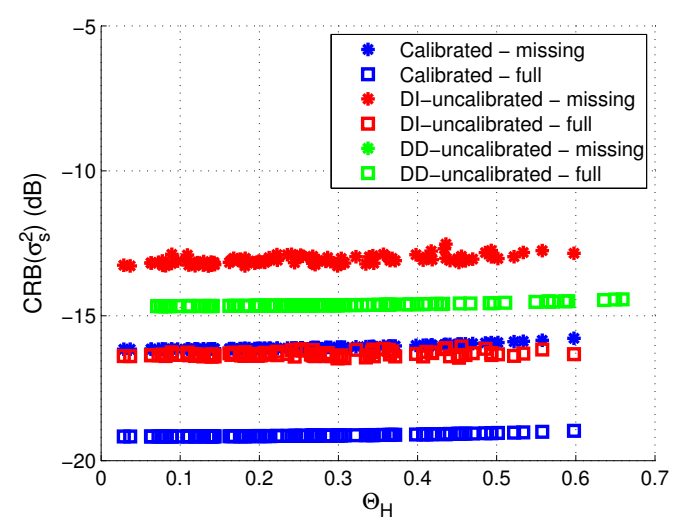

Fig. 2. Evolution of the $\mathrm{CRB}$ of parameter $\sigma_{s}^{2}$ at $\mathrm{SNR}=0 \mathrm{~dB}$ according to the Hermitian angle between the astronomical and RFI subspaces for the calibrated scenario (blue), DI-uncalibrated scenario (red) and DD-uncalibrated scenario (green). The "full data" case is represented by squares, whereas the "missing data" case is represented by stars.

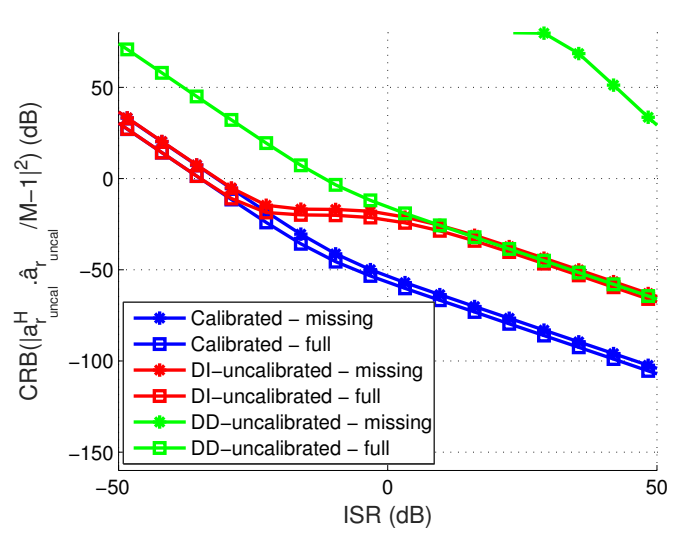

Fig. 3. $\mathrm{CRB}$ of the RFI SSV at SNR $=0 \mathrm{~dB}$ according to the ISR (in $\mathrm{dB}$ ) for the calibrated scenario (blue), DI-uncalibrated scenario (red) and $D D$ uncalibrated scenario (green). The "full data" case is represented by squares, whereas the "missing data" case is represented by stars. 


\section{B. Missing data case}

Most phased array telescopes only access the data covariance $\mathbf{R}_{\mathbf{x x}}{ }^{H}$. In that case, the situation is conceptually similar to a Gaussian interference signal of zero mean and variance $\sigma_{r}^{2}$ (i.e. the signal cyclostationarity is not observed) and the CRB would be given by (5) in which we replace matrix $\mathbf{R}$ by $\mathbf{R}_{\mathbf{x x}}{ }^{H}$, i.e.

$$
\left[\mathbf{P}_{\mathrm{CRB}_{\text {gauss }}}^{-1}\right]_{i, j}=\frac{T}{2} \operatorname{tr}\left(\mathbf{R}_{\mathbf{x x}^{H}}^{-1} \frac{\partial \mathbf{R}_{\mathbf{x x}^{H}}}{\partial \theta_{i}} \mathbf{R}_{\mathbf{x x}^{H}}^{-1} \frac{\partial \mathbf{R}_{\mathbf{x x}^{H}}}{\partial \theta_{j}}\right)
$$

and where parameters $(\omega, \psi)$ are removed from vector $\theta$. This CRB, referred to as $\mathrm{CRB}_{\text {gauss }}$, is used in this paper to investigate the performance loss due to the missing data.

A similar simulation as the one described in section III-A has been conducted, and the results are shown on Figure 1 ("star" data points). A $3 \mathrm{~dB}$ performance loss between the "missing data" and "full data" is observed for the calibrated and DI-uncalibrated scenarios. The astronomical power estimation is not estimable in the DD-uncalibrated scenario as its CRB is far beyond a tolerable estimation error $\operatorname{CRB}_{\text {gauss }}\left\{\sigma_{s}^{2}\right\}_{\text {DD-uncal }}>110 \mathrm{~dB}$ in this simulation, not visible on Figure 1). In that case, the use of the covariance matrix only allows the estimation of the signal subspace but no information distinguishing the two directions $\mathbf{a}_{r_{\text {uncal }}}$ and $\mathbf{a}_{s_{\text {uncal }}}$ is available.

The "star" data points on Figure 2 show the same CRB according to the Hermitian angle between the RFI and astronomical subspaces. The same behavior as in the "full data" case is observed with an approximate $3 \mathrm{~dB}$ performance loss between the calibrated and DI-uncalibrated scenarios, and no estimation possible for the DD-uncalibrated scenario.

On Figure 3, the "star" points represent the CRB of $\mathbf{a}_{r_{\text {uncal }}}$. $\mathrm{A} \approx 3 \mathrm{~dB}$ performance loss between the "full data" and "missing data" cases is again observed for the calibrated and DI-uncalibrated scenarios. The vector estimation is not possible on the ISR range considered in the DD-uncalibrated scenario.

\section{CALIBRATION ERROR IMPACT}

In the previous study we analyzed via the CRB the impact of array calibration on the desired source power and RFI SSV estimation for a known calibration scenario.

In this section, we consider the situation where the considered array model is erroneous. In other words, we assume that the array is well calibrated while it is not. A proper investigation of this situation can be achieved via the use or the "mismatch bound" [19] but would require some tedious derivations that we leave for future works.

Our aim, in this paper, is to illustrate in a simpler way the impact of such calibration error through a basic first order Taylor expansion. For that, consider the case where the estimated power is obtained by the quasi-optimal oblique projector [20] related to the estimated spatial directions $\hat{\mathbf{a}}_{r}=\mathbf{a}_{r}+\delta$ and $\mathbf{a}_{s}$, $\delta$ being an error vector due to the erroneous array model we used.

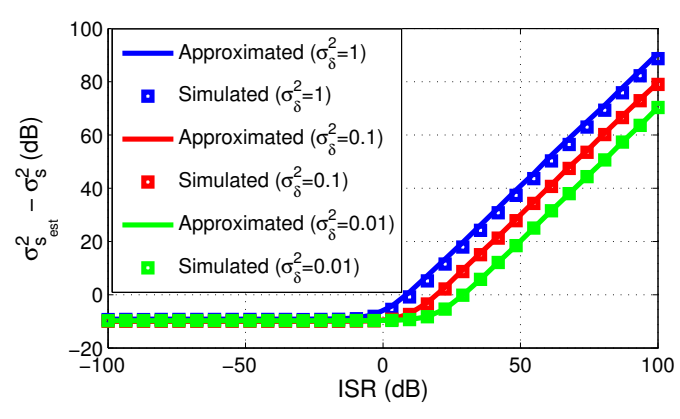

Fig. 4. Error between the approximated and the simulated astronomical source power estimation error at $\mathrm{SNR}=0 \mathrm{~dB}$ as a function of the ISR. The estimation error vector is distributed as $\delta \sim \mathcal{N} \mathcal{C}\left(\mathbf{0}, \sigma_{\delta}^{2} \mathbf{I}\right)$. In blue, $\sigma_{\delta}^{2}=1$. In red, $\sigma_{\delta}^{2}=0.1$. In green, $\sigma_{\delta}^{2}=0.01$.

This oblique projector is expressed as

$$
\mathbf{E}=\mathbf{a}_{s}\left[\mathbf{a}_{s}^{H} \mathbf{P}_{\hat{\mathbf{a}}_{r}} \mathbf{a}_{s}\right]^{-1} \mathbf{a}_{s}^{H} \mathbf{P}_{\hat{\mathbf{a}}_{r}}
$$

and the corresponding power estimator is

$$
\hat{\sigma}_{s}^{2}=\mathbf{a}_{s}^{H} \mathbf{E R E}^{H} \mathbf{a}_{s} /\left\|\mathbf{a}_{s}\right\|^{4}
$$

For simplicity, we assume $\sigma_{r}^{2} \gg \sigma_{n}^{2}$ and $\|\delta\| \ll 1$ in such a way the second order terms of $\delta$ can be neglected. As mentioned earlier, by using a first order Taylor expansion in (10), we obtain

$$
\hat{\sigma}_{s}^{2}=\sigma_{s}^{2}+\frac{\sigma_{n}^{2}}{\left\|\mathbf{a}_{s}\right\|^{2}-|\rho|^{2} /\left\|\mathbf{a}_{r}\right\|^{2}}+\sigma_{r}^{2}|\alpha(\delta)|^{2}
$$

where $\rho=\mathbf{a}_{s}^{H} \mathbf{a}_{r}$ and

$$
\alpha(\delta)=\frac{\rho \mathbf{a}_{r}^{H} \delta}{\left\|\mathbf{a}_{r}\right\|^{2}\left\|\mathbf{a}_{s}\right\|^{2}-|\rho|^{2}}-\frac{\mathbf{a}_{s}^{H} \delta}{\left\|\mathbf{a}_{s}\right\|^{2}-|\rho|^{2} /\left\|\mathbf{a}_{r}\right\|^{2}}
$$

Obviously, contrary to the previous case, the estimation error of $\hat{\sigma}_{s}^{2}$ increases linearly in terms of $\sigma_{r}^{2}$ with a proportionality factor that depends of the direction error vector $\delta$. The latter depends on the calibration error nature which analysis is still under investigation.

Figure 4 compares the approximation of the estimated astronomical power error given in Equation (11) to the error evaluated through Monte-Carlo simulation (1000 trials) according to the ISR. The parameters for this simulation involve an array of $M=10$ antennas, a complex random estimation error vector $\delta \sim \mathcal{N C}\left(\mathbf{0}, \sigma_{\delta}^{2} \mathbf{I}\right), \sigma_{s}^{2}=\sigma_{n}^{2}=1$ and the astronomical and RFI SSV are complex vectors made of random phases only (unit gain for each vector entry) uniformly distributed over $[0,2 \pi]$.

As depicted on the Figure, the astronomical power error significantly increases with the interference power for ISR $>0$ $\mathrm{dB}$ even for low RFI SSV estimation errors, i.e. for $\sigma_{\delta}^{2}=0.01$.

\section{RESUlts ANALYSiS}

Figures 1 and 2 present a numerical analysis of the CRB of the estimate of an astronomical source power impinging a phased array made of 10 antennas. The following comments can be made: 
- Uncalibration has an important impact for source localization [21].

- Array uncalibration can also have a significant impact in source power recovery in presence of interfering signals.

- The best source power estimation is achieved for perfectly calibrated arrays.

- In case of direction-independent calibration, the source estimation performance increases as the interference dominates both the astronomical signal and the system noise, allowing more accurate RFI mitigation.

- Source recovery is not possible in case of missing data when the array exhibits a direction-dependent uncalibration $\left(\mathrm{CRB}_{\text {gauss }}\left\{\sigma_{s}^{2}\right\}_{\text {DD-uncal }}>110 \mathrm{~dB}\right.$ in our simulation) as no RFI isolation can be made with the data available.

- The "missing data" case in general prevents from the exploitation of the RFI cyclostationary and non-circular properties. These properties allow a better SSV estimation performance as they are not shared with the astronomical source nor the system noise [10], [11]. Neglecting this information translates into poor RFI mitigation performance (compared to cyclostationary- or noncircular-based approaches), and hence lower astronomical power estimation performance. A similar observation is expected for non-Gaussian interference. A $3 \mathrm{~dB}$ performance loss is noted from the simulation between the "full data" case and the "missing data" case.

- The impact of array uncalibration on astronomical source power recovery can however become significant (see illustration of Figure 4) in case of data model misspecification [19]. Further analysis are required to evaluate the impingement of calibration model errors for astronomical sources estimation.

\section{CONCLUSION}

This paper analyzes the performance of a statistically optimum unbiased estimator of an astronomical source power impinging a phased array radio telescope concurrently with a cyclostationary non-circular interference and in presence of system noise. Particularly, this analysis considers three array calibration scenarios : perfectly calibrated, direction-independent uncalibrated and direction-dependent uncalibrated.

As can be expected, the perfect array calibration scenario presents the best estimation performance, independently of the ISR. Direction-independent uncalibration allows similar performance when the astronomical source and the system noise become negligible compared to the RFI. Direction-dependent uncalibration induces the poorest performance when the extended covariance matrix is disregarded, and does not allow a source power recovery without considering the cyclic characteristics of the interference. A $3 \mathrm{~dB}$ loss is observed as compared to the "full data" case, in the DI-uncalibrated and calibrated scenarios.

We conjecture that this performance loss might be even higher when other communication signal properties (nonGaussianity with finite alphabet, time correlation due to oversampling) are taken into account.
Calibration modeling errors affect more severely the estimation performance, especially for large ISR, and are illustrated here by a simplified but useful first order error derivation. Further analysis of the "mismatch" Cramér-Rao bound will allow the quantification of the loss of performance in case of erroneous calibration assumption.

\section{REFERENCES}

[1] K.F. Warnick, R. Maaskant, M.V. Ivashina, D.B. Davidson, and B.D. Jeffs, "High-sensitivity phased array receivers for radio astronomy," Proceedings of the IEEE, vol. PP, no. 99, pp. 1-16, 2016.

[2] K.F. Warnick, B.D. Jeffs, J. Landon, J. Waldron, D. Jones, J.R. Fisher, and R. Norrod, "Beamforming and imaging with the byu/nrao 1band 19-element phased array feed," in Antenna Technology and Applied Electromagnetics and the Canadian Radio Science Meeting, 2009. ANTEM/URSI 2009. 13th International Symposium on, Feb 2009, pp. $1-4$.

[3] A. Chippendale, J. O’Sullivan, J. Reynolds, R. Gough, D. Hayman, and S. Hay, "Phased array feed testing for astronomy with askap," in Phased Array Systems and Technology (ARRAY), 2010 IEEE International Symposium on, Oct 2010, pp. 648-652.

[4] W.A. van Cappellen, L. Bakker, and T.A. Oosterloo, "Experimental results of the apertif phased array feed," in General Assembly and Scientific Symposium, 2011 XXXth URSI, Aug 2011, pp. 1-4.

[5] W.A. Baan, "Rfi mitigation in radio astronomy," in General Assembly and Scientific Symposium, 2011 XXXth URSI, Aug 2011, pp. 1-2.

[6] M.J. Bentum and A.J. Boonstra, "Low frequency astronomy 2014; the challenge in a crowded rfi environment," in General Assembly and Scientific Symposium, 2011 XXXth URSI, Aug 2011, pp. 1-4.

[7] B.D. Jeffs, K. Warnick, and L. Li, "Improved interference cancellation in synthesis array radio astronomy using auxiliary antennas," in Acoustics, Speech, and Signal Processing, 2003. Proceedings. (ICASSP '03). 2003 IEEE International Conference on, April 2003, vol. 5, pp. V-77-80 vol.5.

[8] S. van der Tol and A.J. van der Veen, "Performance analysis of spatial filtering of $\mathrm{rf}$ interference in radio astronomy," Signal Processing, IEEE Transactions on, vol. 53, no. 3, pp. 896-910, March 2005.

[9] A.J. Boonstra, Radio frequency interference mitigation in radio astronomy, TU Delft, Delft University of Technology, 2005.

[10] W.A. Gardner, "Cyclostationarity in communications and signal processing," Tech. Rep., DTIC Document, 1994.

[11] B. Picinbono, "On circularity," Signal Processing, IEEE Transactions on, vol. 42, no. 12, pp. 3473-3482, Dec 1994.

[12] G. Hellbourg, T. Trainini, R. Weber, E. Moreau, C. Capdessus, and A.J. Boonstra, "Rfi subspace estimation techniques for new generation radio telescopes," in Signal Processing Conference (EUSIPCO), 2012 Proceedings of the 20th European, Aug 2012, pp. 200-204.

[13] B.D. Jeffs and K.F. Warnick, "Spatial array processing methods for radio astronomical rfi mitigation," in Radio Science Meeting (USNCURSI NRSM), 2013 US National Committee of URSI National, Jan 2013.

[14] S.J. Wijnholds, S. van der Tol, R. Nijboer, and A.J. van der Veen, "Calibration challenges for future radio telescopes," Signal Processing Magazine, IEEE, vol. 27, no. 1, pp. 30-42, Jan 2010.

[15] A.J. Boonstra, A.J. van der Veen, and J. Raza, "Spatial filtering of continuous interference in radio astronomy," in Acoustics, Speech, and Signal Processing (ICASSP), 2002 IEEE International Conference on, May 2002, vol. 3, pp. 2933-2936.

[16] P. Stoica and R.L. Moses, Spectral analysis of signals, Pearson/Prentice Hall Upper Saddle River, NJ, 2005.

[17] G. Hellbourg, R. Weber, K. Abed-Meraim, and A. J. Boonstra, "Rfi spatial processing at nancay observatory : Approaches and experiments," in 2014 IEEE International Conference on Acoustics, Speech and Signal Processing (ICASSP), May 2014, pp. 5387-5391.

[18] K. Scharnhorst, "Angles in complex vector spaces," Acta Applicandae Mathematica, vol. 69, no. 1, pp. 95-103, 2001.

[19] C.D. Richmond and L.L. Horowitz, "Parameter bounds under misspecified models," in Signals, Systems and Computers, 2013 Asilomar Conference on, Nov 2013, pp. 176-180.

[20] G. Hellbourg, Radio Frequency Interference spatial processing for modern radio telescopes, Ph.D. thesis, Université d'Orléans, 2014.

[21] A. Boudjellal, Contributions to source localization and separation, Ph.D. thesis, Université d'Orléans, 2015. 